\title{
Evaluation of the surface structure of composite restorations in light of own pilot research
}

\author{
Renata Chalas ${ }^{1 *}$, Miroslaw Orlowski ${ }^{1}$, Bozena Tarczydlo ${ }^{1}$, \\ JoAnna Zubrzycka-Wrobel ${ }^{1}$, Karol Maj $^{2}$, Ilona WojCiK-Checinska ${ }^{1}$
}

${ }^{1}$ Chair and Department of Conservative Dentistry and Endodontics, Medical University of Lublin, Karmelicka 7, 20-081 Lublin, Poland ${ }^{2}$ Dental Clinical Center II, Lubartowska 58, 20-094 Lublin, Poland

\section{ARTICLE INFO}

Received 10 May 2016

Accepted 16 June 2016

\section{Keywords:}

dental composites,

surface assessment,

$3 \mathrm{D}$ analysis.

\begin{abstract}
Research on the dental restorative materials employed in remedying dental cavities has been conducted on many levels and areas, both with application of clinical and laboratory methods. One of the elements that determines whether the restoration may be degraded is the condition of its surface. The aim of the study was to assess the texture surface of composite restorations using a non-contact method of teeth models scanning. In this work, ten medium size cavities on the occlusal surfaces of molars in adult patients were prepared and restored with resin composite. Before undertaking the procedure and after the finishing and polishing of the restorations, impressions were taken and sent into the laboratory so as to prepare plaster casts. Every cast was then scanned utilizing the noncontact 3D surface measurement instrument so as to assess the texture surface of the restoration. The resulting three dimensional analyses of post-restoration models showed the correct marginal adaptation of resin composite dental material to the hard tooth structures and its smooth filling occlusal surface. Additional comparison of scans done before and after restoring the cavities allowed the calculating of differences in volume, mean and maximum heights. The applied method of analysis is thought to be helpful in the detailed evaluation of restoration dental material texture. Moreover, the enabled possibility of continuous observation is expedient for assessing the usefulness of the method in standard dental practice.
\end{abstract}

\section{INTRODUCTION}

Composite materials are currently the most widely and frequently used biomaterials in dentistry. Reconstructive dental biomaterials, thanks to modern technologies, more and more precisely imitate the restored mineralized tooth tissues, both in terms of aesthetics and biofunctionality. The dental industry provides a wide range of different restorative materials, and we expect them to meet all or most of the set requirements. Still, the quality of the restoration is a sum of many factors. Among these are the physical-chemical characteristics of the material, the condition of the tooth tissues, cavity preparation, the manual skills and knowledge of the attending dentist, as well as the commitment and willingness to cooperate on the part of the patient.

Research on dental restorative materials used for dental cavities has been conducted on many levels and areas, and evidences the application of clinical and laboratory methods.

\footnotetext{
* Corresponding author

e-mail: renata.chalas@umlub.pl
}

Among clinical criteria used in overviewed literature, it was found that Ryge's scale is still very common in clinical conditions for assessing the following parameters of restorations: the surface structure (criteria: color, smoothness, gloss, discoloration); anatomical shape (criteria: restoration of anatomical shape, nodules and shear surface); marginal adhesion (criteria: the presence of marginal fissure, discoloration, cracks, damage to the edges of the filling, secondary caries). With regard to such parameters, the numerical Ryge's scale allows for a comparison of the quality of restoration in the subsequent control examinations and the tracking of its changes over time [3]. In laboratory studies, other exploratory methods are used as marginal integrity assessment in materials engineering or chemical engineering $[7,10,12]$, and it should be underlined that the on-going development of dental technology allows the use of modern equipment and technologies for in-depth analysis of the parameters of interest. In such work, atomic force microscopy has been used in studies of geometric structure and the physical condition of the surface [4]. The atomic force 
microscope (AFM) enables imaging of a surface in three dimensions, with a resolution of up to nanometers. AFM is a tool providing direct, extremely precise contact with the tested material. It enables a scanning of materials surface structures that are in a wide range of hardness. Moreover, it allows the obtaining of images with exceptional precision, which, in turn, enables an in-depth analysis of the test surface $[1,4]$. Furthermore, in current literature, studies can be found on surface properties that come about by way of using profilometry and inverse gas chromatography techniques $[1,15]$.

One of the elements that determines whether the restoration may be degraded is the condition of its surface. The restored surface reproduces (imitates) the anatomical surface of a natural tooth, and therefore is exposed to the number of physical, chemical and biological factors acting on the tooth in the mouth $[8,9]$. Herein, the smooth, polished surface of composite dental restorations protects the tooth tissues from the attack of cariogenic factors, while the roughness of the surface of the filler material increases the accumulation of bacterial biofilm [11,14].

The aim of the study was to assess the texture surface of composite restorations using a non-contact method of teeth models scanning.

\section{MATERIAL AND METHODS}

Ten medium size cavities on the occlusal surfaces of molars in adult patients were prepared and restored with microhybrid composite. Before the procedure and after the finishing and polishing of the restorations, precise impressions were taken and sent into the laboratory so as to prepare plaster casts. Every cast was scanned using the Proscan 2000 non-contact 3D surface measurement instrument (Scantron Inustrial Products Limited, United Kingdom) so as to assess the texture surface of the restoration. The technology is based on the employment of a confocal multiplexing sensor with up to $0.01 \mu \mathrm{m}$ resolution, and additional using of the ProForm software enabled a comparison of two scans. This is a non-contact approach for measuring surfaces sensitive to the influence of a contact technology, and it is devoid of problematic penetration (a defect previously observed when using laser displacement techniques). It works by transmitting safe white light through a lens. This light is then divided into the full spectral field, focusing each of the different colour frequencies at a slightly different point through a defined measuring range. When an object is placed within this range, only one particular color frequency is reflected back from the surface. This information is then passed back into a processor where a spectrometer analyses the signal and converts it to a measurement. The Proscan combines these measurements with the precise location of a moving $\mathrm{X} / \mathrm{Y}$ linear table, creating three co-ordinates from which to create a three dimensional profile.

\section{RESULTS}

Scanning of the surfaces of the tooth models created prior to the undertaken procedures allowed seeing the depth of the cavity via three dimensional analyses, while the post-scanning of post-restoration models showed the correct marginal adaptation of microhybrid resin composite dental material to the hard tooth structures. 3D surface measurement of composite also revealed its smooth filling of the occlusal surface. Additional comparison of scans done before and after cavities restoration allowed calculating differences in volume, mean and maximum heights. Chosen scans of surfaces of composite restorations are presented in Fig. 1-4.

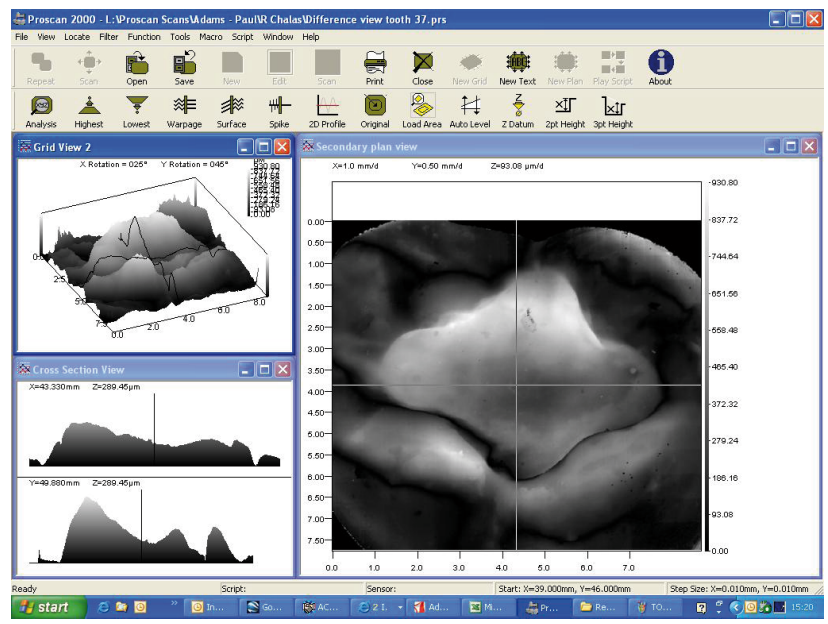

Figure 1. Three dimensional view of tooth 37 before restoration

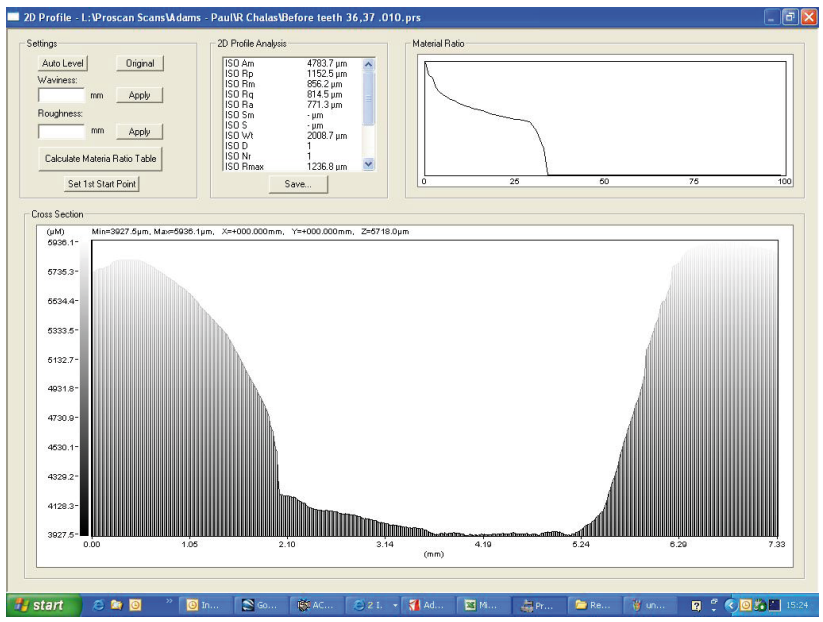

Figure 2. Cross-section of the occlusal surface of tooth 37

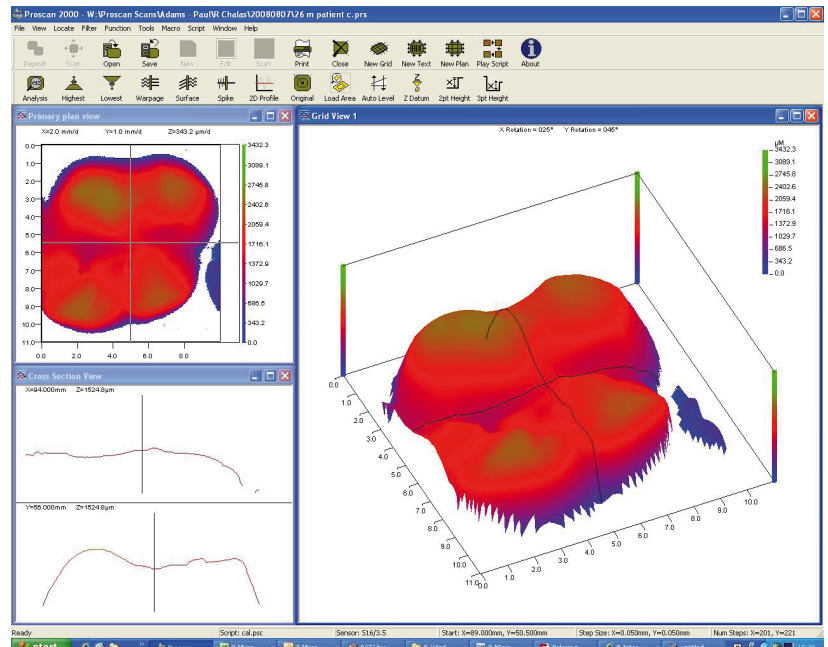

Figure 3. The occlusal surface of the surface of composite restoration 


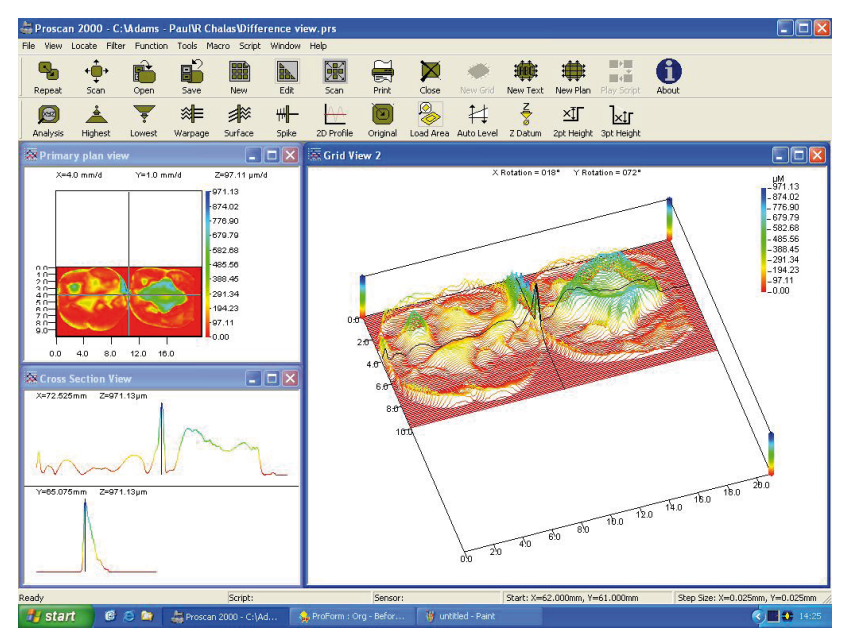

Figure 4. Differences between before and after restoration for tooth 36 and 37 (line version)

\section{DISCUSSION}

The complexity of the phenomena occurring in the mouth justifies the need for scientific research of biomaterial properties [2], and introduction of any new material to the market requires both experimental and clinical trials. The latter are usually complementary to the laboratory tests necessary for obtaining a full assessment of the material. Herein, the maintenance of the restoration in the mouth and the smoothness of its surface are undoubtedly the most important factors evaluated [3]. Under natural physiological conditions, a multiplicity and complexity of external factors (load, temperature) and internal factors (saliva, the condition of the tooth tissue) influence the wear of teeth and dental materials [13]. The quality of the composite material surface impacts upon the durability of restoration, and indirectly the condition of the tissues surrounding the restoration - both of the enamel, dentin, and the mucosa. Furthermore, the smoothness of the polished surface of the composite allows for the relative protection of the surrounding tissues against the attack of cariogenic bacteria that may colonize the surface [11]. The roughness of the surface depends on the size of the filler particles and the quality of the connection to the resin matrix [14], while the surface quality achieved during polishing depends, inter alia on the resin composition constituting the polished composite $[4,13,15]$. of note, a thoroughly polymerized resin within any composite significantly effects the reduction of discoloration because the dyes are deposited mainly in its structure [5]. In the present paper, a microhybrid composite has been used. This presents a reduced proneness to the deposition of dyes and has a very good polishability, and, thus, smoothness of the surface [6]. The application of the presented method of indirect assessment of composite dental restorations surface seems to be an interesting solution that combines the advantages of in-vitro tests (scanning of models outside the patient's mouth) and in-vivo tests (obtaining the clinical picture of the oral cavity through accurate impression and model). The precision of scanning with the accuracy up to 0.01 microns allows for a very precise analysis of the assessed surface of restoration. This is extremely important in predicting the durability of dental materials.

\section{CONCLUSIONS}

Non-contact 3D surface analysis is helpful in evaluating dental material condition. The applied method also allows the revealing of the surface texture details of restorations. What is more, the allowed possibility of continuous observation is expedient for assessing the usefulness of the method in standard dental practice.

\section{REFERENCES}

1. Batko K. et al.: Characteristics of the surface layer of selected glassionomer cements by inverse gas chromatography in increased humidity conditions. Polimery, 51, 280, 2006.

2. Chałas R. et al.: Assessment of dentin reaction after Biodentine application. Curr. Iss. Pharm. Med. Sci., 26, 435, 2013.

3. Chałas R. et al.: Long-term clinical performance of experimental glass-ionomer cement with the bi-functional fluid. J. Pre-Clin. Clin. Res., 9, 109, 2015.

4. Jamróz-Wilkońska L., Wilkoński W., Krupiński J.: Evaluation of surface roughness of composite materials using an Atomic Force Microscope (AFM) after polishing with different polishing systems. Mag. Stomat., 26, 144, 2014.

5. Jamróz-Wilkońska L., Wilkoński W., Krupiński J.: The spectrophotometric evaluation of surface discolourations of composite materials after polishing with different polishing systems. e-Dentico, 35, 70, 2012.

6. Li Y. et al.: Effect of filler content and size on properties of composites. J. Dent. Res., 64, 1396, 1985.

7. Łagan S.: Estimation of possibility of application of CIMM technique for direct composite fillings in the $1^{\text {st }}$ and $2^{\text {nd }}$ class cavities according to Black. Akt. Probl. Biomech., 3, 131, 2009.

8. Mystowska J., Dąbrowski J.R.: Tribological characteristics of kinematics couple: tooth-composite material for permanent dental fillings. Ekspl. i Niezawod. - Maint. and Reliabil., 12, 4, 2010.

9. Nagarajan V.S., Jahanmir S., Thompson V.P.: In vitro contact wear of dental composites. Dent. Mater., 20, 63, 2004.

10. Olek A., Klimek L., Bołtacz-Rzepkowska E.: Research methods of material engineering used in dental experimental studies. Mag. Stomat., 17, 66, 2015.

11. Ono M. et al.: Surface properties of resin composite materials relative to biofilm formation. Dent. Mater. J., 26, 613, 2007.

12. Orłowski M., Tarczydło B., Chałas R.: Evaluation of marginal integrity of four bulk-fill dental composite materials: in vitro study. Sci. World J., 2015; 2015:701262. doi: 10.1155/2015/701262. Epub 2015 Mar 22.

13. Turssi C.P., Rodrigues A.L. Jr., Serra M.C.: Textural characterization of finished and polished composites over time of intraoral exposure. J. Biomed. Mater. Res. B Appl. Biomater., 76, 381, 2006.

14. Watanabe $T$. et al. Influence of polishing duration on surface roughness of resin composites. J. Oral Sci., 47, 21, 2005.

15. Yazici A.R., Muftu A., Kugel G.: Three-dimensional surface profile analysis of different types of flowable restorative resins following different finishing protocols. J Contemp. Dent. Pract., 5, 9, 2007. 